\title{
Small-x Scattering and Gauge/Gravity Duality
}

\author{
Miguel Costa, Marko Djurić* \\ University of Porto \\ E-mail: miguelc@fc.up.pt,djuricefc.up.pt
}

\section{Nick Evans}

University of Southampton

E-mail: n.j.evansesoton.ac.uk

\begin{abstract}
We show how scattering at small-x can be studied by Pomeron exchange in AdS space using the gauge/gravity duality, with good results. In this approach the Pomeron at strong coupling is the graviton Regge trajectory. We use AdS space with a hard wall to mimic confinement effects. We use our results to fit HERA data, most recently for vector meson production, where both the proton and vector mesons are described by simple holographic wavefunctions in AdS. Our previous fits to deep inelastic scattering and deeply virtual Compton scattering data will also be briefly discussed.
\end{abstract}

XXII. International Workshop on Deep-Inelastic Scattering and Related Subjects, 28 April - 2 May 2014

Warsaw, Poland

${ }^{*}$ Speaker. 


\section{Introduction}

HERA is the largest electron - proton collider ever built, and has tremendously improved our knowledge of the structure of the proton. It operated from 1992-2007, and collected a wealth of small $x$ data. Two of the crucial discoveries important for these proceedings are that the cross sections for many different processes (DIS, DVCS, VM production...) show a power growth with $1 / x$ and that the same, universal gluon distribution functions describe these processes, and gluons dominate at small $x$. These point to a universal Pomeron exchange as the dominant process. Traditionally Pomeron exchange can be studied using the perturbative BFKL approach. Our goal is to apply an alternative method to study the non-perturbative and saturation regions, and also see how much can they be applied to the higher $Q^{2}$ region as well.

\section{Pomeron in AdS}

The Pomeron is the leading order exchange in all total cross sections, and in $2 \rightarrow 2$ amplitudes with the quantum numbers of the vacuum, in the Regge limit $s \gg t$. It is the sum of an infinite number of states with the quantum numbers of the vacuum, and the tell-tale sign of Pomeron exchange is that it leads to an amplitutude that asymptotically, as $s \rightarrow \infty$, goes as

$$
A(s, t) \sim s^{\alpha(t)}, \alpha(t)=\alpha(0)+\frac{\alpha^{\prime} t}{2} .
$$

In perturbative $\mathrm{QCD}$, the propagation of the Pomeron is given by the BFKL equation. However, we will use an approach that is based on the AdS/CFT correspondence [1]. This is a conjectured exact duality between type IIB string theory on $A d S_{5} \times S_{5}$, and $\mathscr{N}=4$ SYM, on the boundary of $A d S_{5}$. Since QCD at low $x$ has a lot of similarities with $\mathscr{N}=4 \mathrm{SYM}$, this duality is very useful. It relates states in string theory to operators in the field theory through the relation

$$
\left\langle e^{\int d^{4} x \phi_{i}(x) \mathscr{O}_{i}(x)}\right\rangle_{C F T}=\mathscr{Z}_{\text {string }}\left[\left.\phi_{i}(x, z)\right|_{z \sim 0}\right] .
$$

The metric of $A d S_{5}$ space can be expressed in several forms. The one most useful for our purposes is

$$
d s^{2}=e^{2 A(z)}\left[-d x^{+} d x^{-}+d x_{\perp} d x_{\perp}+d z d z\right]+R^{2} d^{2} \Omega_{5} .
$$

The exponent

$$
2 A(z)=\frac{R^{2}}{z^{2}}
$$

where $z$ is the extra radial coordinate of $A d S$ space. It is possible to study theories where this factor is different, and only asymptotically reduces to this form, in order to obtain a theory which more closely mimics QCD, for example by introducing a scale for confinement. These theories in general are referred to as 'gauge/gravity duality'. Possibly the simplest such model is the so-called 'hard-wall' model, where the metric takes the form (2.3) with $A(z)$ given by (2.4) only up to some sharp cut-off $z_{0} \sim 1 / \Lambda_{Q C D}$, setting the scale for confinement. This is the model we will use, in addition to the conformal model (2.4), in the fits below. The correspondence works in the limit

$$
N_{C} \rightarrow \infty, \lambda=g^{2} N_{C}=R^{4} / \alpha^{\prime 2} \gg 1, \text { fixed }
$$


making it useful for exploring the non-perturbative region where the coupling $\lambda$ is large. Understandably there is not enough space in these proceedings to explain this correspondence, and the interested reader is directed to one of the many reviews, e.g. [2]. More details on using the hardwall model can be found in [3] and references therein. Note that although it has not been proven, there is a lot of evidence for this correspondence, for example 2 and 3 point functions that are independent of $\lambda$ have been calculated from both sides and shown to match. We can calculate scattering amplitudes by using Witten diagrams, which are analogous to Feynman diagrams. In order to use them to study Pomeron exchange, we therefore need to first calculate its propagator in $A d S$. As shown in [4], the Pomeron in AdS string theory is the Regge trajectory of the graviton. It is given by

$$
\chi(\tau, L)=\left(\cot \left(\frac{\pi \rho}{2}\right)+i\right) g_{0}^{2} e^{(1-\rho) \tau} \frac{L}{\sinh L} \frac{\exp \left(\frac{-L^{2}}{\rho \tau}\right)}{(\rho \tau)^{3 / 2}},
$$

with $\tau=\log \left(z z^{\prime} s / \sqrt{\lambda}\right), L=\log (1+v+\sqrt{v(2+v)})$ and $v$ the chordal distance in $H_{3}$. Similar procedure can be applied for other trajectories, e.g. for the Odderon [5]. In order to satisfy the Froissart bound, we need to take into account effects beyond single Pomeron exchange. One way is to use the eikonal approximation, which sums Pomeron exchanges to all orders, but ignores the interactions between them [6]

$$
A(s, t)=2 i s \int d^{2} b e^{-i \mathbf{b} \cdot \mathbf{q} \perp} \int d z d \bar{z} P_{13}(z) P_{24}(\bar{z})\left(1-e^{i \chi(s, b, z, \bar{z})}\right) .
$$

We can study different scattering processes by supplying $P_{13}$ and $P_{24}$, which are the wavefunctions in $A d S$ for the external scattering states. For example, this has already been applied to DIS[3, 7], and DVCS [8].

\section{Vector Meson Production}

Vector meson production occurs in the scattering between an offshell photon and a proton. The vector mesons consist of a quark-antiquark pair, and have the same quantum numbers as the photon, $J^{P C}=1^{--}$. The production of the $\rho^{0}, \omega, \phi$ and $J / \Psi$ was measured at HERA [9]. We are interested in calculating the differential and exclusive cross sections

and

$$
\frac{d \sigma}{d t}\left(x, Q^{2}, t\right)=\frac{|W|^{2}}{16 \pi s^{2}},
$$

$$
\sigma\left(x, Q^{2}\right)=\frac{1}{16 \pi s^{2}} \int d t|W|^{2} .
$$

Here $W$ is the scattering amplitude

$$
W=2 i s Q Q^{\prime} \int d l_{\perp} e^{i q_{\perp} \cdot l_{\perp}} \int \frac{d z}{z^{3}} \frac{d \bar{z}}{\bar{z}^{3}} \Psi(z) \Phi(\bar{z})\left[1-e^{i \chi(S, L)}\right] .
$$

This has the previously mentioned form, we just need to supply the wavefunctions $\Psi(z)$ and $\Phi(\bar{z})$ for the photon and the proton. In this analysis we use

$$
\Psi_{n}(z)=-\left(\sqrt{C \frac{\pi^{2}}{6}} z^{2} K_{n}(Q z)\right)\left(\frac{\sqrt{2}}{\xi J_{1}(\xi)} z^{2} J_{n}(m z)\right), \Phi(\bar{z})=\bar{z}^{3} \delta\left(\bar{z}-z_{\star}\right) .
$$

The different vector mesons are differentiated above by their mass $m$. For more on how these were obtained see [10], and for more information on vector mesons in gauge/gravity duality see [11]. 


\section{Models}

We start with the conformal pomeron, with

$$
1-e^{i \chi} \approx-i \chi=-i\left(\cot \left(\frac{\pi \rho}{2}\right)+i\right) g_{0}^{2} e^{(1-\rho) \tau} \frac{L}{\sinh L} \frac{\exp \left(\frac{-L^{2}}{\rho \tau}\right)}{(\rho \tau)^{3 / 2}} .
$$

It depends on 3 parameters, $z_{*}$ which is related to the size of the target, $\rho=2-j_{0}=2 / \sqrt{\lambda}$ and $C g_{0}^{2}$ which depends on the coupling of the Pomeron to external states. We will also use the hard-wall Pomeron model. First notice that at $t=0$ (which is enough for DIS [3]) $\chi$ for conformal pomeron exchange can be integrated in impact parameter

$$
\chi(\tau, t=0, z, \bar{z})=i \pi g_{0}^{2}\left(\cot \left(\frac{\pi \rho}{2}\right)+i\right)(z \bar{z}) e^{(1-\rho) \tau} \frac{e^{-\frac{(\ln (\bar{z} / z))^{2}}{\rho \tau}}}{(\rho \tau)^{1 / 2}} .
$$

Similarly, the $t=0$ result for the hard-wall model can also be written explicitly

$$
\chi_{h w}(\tau, t=0, z, \bar{z})=\chi(\tau, 0, z, \bar{z})+\mathscr{F}(\tau, z, \bar{z}) \chi\left(\tau, 0, z, z_{0}^{2} / \bar{z}\right) .
$$

When $t \neq 0$, we will use an approximation

$$
\chi_{h w}(\tau, l, z, \bar{z})=C(\tau, z, \bar{z}) D(\tau, l) \chi_{h w}^{(0)}(\tau, l, z, \bar{z}),
$$

for details see [10,3]. The function

$$
\mathscr{F}(\tau, z, \bar{z})=1-4 \sqrt{\pi \tau} e^{\eta^{2}} \operatorname{erfc}(\eta), \quad \eta=\frac{-\log \left(z \bar{z} / z_{0}^{2}\right)+4 \tau}{\sqrt{4 \tau}}
$$

is set by the boundary conditions at the wall and represents the relative importance of the two terms, and hence the importance of confinement. It varies between -1 and 1 , approaching -1 at either large $z$, which roughly corresponds to small $Q^{2}$, or at large $\tau$ corresponding to small $x$.

\section{Data Analysis}

For vector meson production, we fit the data collected at HERA by the $\mathrm{H} 1$ experiment [9]. All the data is at small $x(x<0.01)$, where Pomeron exchange is the dominant process. We will look at both the differential and total exclusive cross sections. Our results are summarized in figure 1 taken from [10], where we direct the reader for further details, discussion and figures. The fits to the full cross-sections provide very good $\chi^{2}<1$ in all cases. The fits for the differential cross section are less good but still have $\chi^{2}<2$ in each case. To claim such a good fit for the $J / \Psi$ meson we do need to include the hard-wall parameter $z_{0}$ and this is the only place in our fits where it makes a significant impact. Note that the same formalism has been applied before to DIS with good results $\left(\chi^{2}=1.04\right.$ for the best model) [3, 7], and DVCS $\left(\chi^{2}=1.00\right.$ and $\chi^{2}=0.51$ for the best models of the cross section and differential cross section respectively) [8]. In particular the DIS data fit in [3] probes a kinematical region where confinement is more important, and a more significant difference between the conformal and hard wall models was obtained. In the same reference the effective running of the Pomeron intercept was calculated. 


\begin{tabular}{|c|c|c|c|c|c|c|c|c|}
\hline & \multicolumn{4}{|c|}{$\sigma[\mathrm{nb}]$} & \multicolumn{3}{|c|}{$\mathrm{d} \sigma / \mathrm{dt}\left[\mathrm{nb} / \mathrm{GeV}^{2}\right]$} \\
\hline & & $\rho$ & $\phi$ & $\Omega$ & $J / \psi$ & $\rho$ & $\phi$ & $J / \psi$ \\
\hline & m [GeV] & 0.77549 & 1.019445 & 0.78265 & 3.096916 & 0.77549 & 1.019445 & 3.096916 \\
\hline & $\mathrm{N}$ & 48 & 27 & 6 & 38 & 35 & 21 & 84 \\
\hline \multirow{4}{*}{$\begin{array}{l}C \\
0 \\
n \\
f \\
o \\
r \\
m \\
a \\
l\end{array}$} & $\chi^{2}$ & 0.92 & 0.60 & 0.0099 & 0.28 & 1.7 & 1.3 & 2.9 \\
\hline & $g_{0}^{2}$ & 4.6 & 1.8 & 0.53 & 0.62 & 1.6 & 0.25 & 0.56 \\
\hline & $\rho$ & 0.76 & 0.73 & 0.64 & 0.70 & 0.65 & 0.54 & 0.72 \\
\hline & $z^{*}\left[\mathrm{GeV}^{-1}\right]$ & 3.4 & 3.0 & 1.8 & 0.98 & 2.1 & 2.5 & 2.2 \\
\hline \multirow{5}{*}{$\begin{array}{l}\mathrm{H} \\
\mathrm{a} \\
\mathrm{r} \\
\mathrm{d} \\
\mathrm{w} \\
\mathrm{a} \\
\mathrm{l} \\
\mathrm{l}\end{array}$} & $\alpha^{2}$ & 0.88 & 0.61 & 0.015 & 0.30 & 1.7 & 1.4 & 1.8 \\
\hline & $g_{0}^{2}$ & 4.1 & 1.8 & 0.67 & 0.75 & 2.2 & 0.38 & 0.69 \\
\hline & $\rho$ & 0.76 & 0.73 & 0.66 & 0.71 & 0.69 & 0.59 & 0.75 \\
\hline & $z^{\star}\left[\mathrm{GeV}^{-1}\right]$ & 3.6 & 3.6 & 1.5 & 0.87 & 2.2 & 2.5 & 2.4 \\
\hline & $z_{0}\left[\mathrm{GeV}^{-1}\right]$ & 4.8 & 4.4 & 7.3 & 5.3 & 7.7 & 8.6 & 4.6 \\
\hline
\end{tabular}

Figure 1: Fit results.

\section{Conclusion}

We have seen some interesting methods for applying gauge/gravity duality to study small $x$ physics. In particular we can study a wide range of HERA processes, and in kinematical regions not accessible by traditional methods. Some of the data show that we need our theory to include confinement if we want it to be realistic, as we can see in figure 2. More work is needed to see the generality of this result. We now have 3 processes (DIS, DVCS and vector meson production)

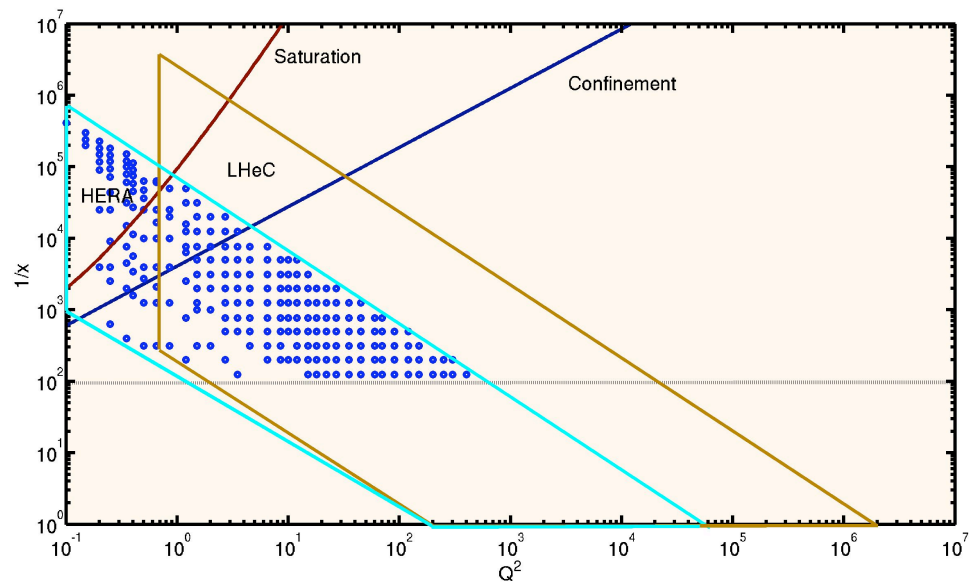

Figure 2: In our model the importance of confinement enters earlier than saturation. 
where the AdS (BPST) pomeron exchange gives excellent agreement with experiment in the strong coupling region, and even up to relatively high $Q^{2}$. Hence string theory on $\operatorname{AdS}$ is giving us interesting insights into non-perturbative scattering. The value of the pomeron intercept is in the region $1.2-1.4$ which is in the crossover region between strong and weak coupling, and a lot of the equations have a form which is very similar both at weak and at strong coupling (but $\chi$ is different). It might therefore be possible to extend some of the insights we gain even into the weak coupling regime. It is also interesting to extend these methods beyond $2 \rightarrow 2$ scattering (e.g. for $2 \rightarrow 3$ scattering [12]). Eventually it would be good to have a single set of parameters that fits several different processes.

\section{Acknowledgments}

This work was partially funded by grants PTDC/FIS/099293/2008 and CERN/FP/116358/2010. Centro de Física do Porto is partially funded by FCT. The work of M.D. is supported by the FCT/Marie Curie Welcome II program. N.E. is grateful for the support of an STFC rolling grant. The research leading to these results has received funding from the People Programme (Marie Curie Actions) of the European Union's Seventh Framework Programme FP7/2007-2013/ under REA Grant Agreements No 269217 and No 317089.

\section{References}

[1] J. M. Maldacena, Adv. Theor. Math. Phys. 2 (1998) 231 [hep-th/9711200]. E. Witten, Adv. Theor. Math. Phys. 2 (1998) 253 [hep-th/9802150].

[2] O. Aharony, S. S. Gubser, J. M. Maldacena, H. Ooguri and Y. Oz, Phys. Rept. 323 (2000) 183 [hep-th/9905111]. J. Erdmenger, Lect. Notes Phys. 851 (2012) 99.

[3] R. C. Brower, M. Djuric, I. Sarcevic and C. -ITan, JHEP 1011 (2010) 051 [arXiv:1007.2259 [hep-ph]].

[4] R. C. Brower, J. Polchinski, M. J. Strassler and C. -ITan, JHEP 0712 (2007) 005 [hep-th/0603115].

[5] R. C. Brower, M. Djuric and C. -ITan, JHEP 0907 (2009) 063 [arXiv:0812.0354 [hep-th]].

[6] L. Cornalba, M. S. Costa, J. Penedones and R. Schiappa, JHEP 0708 (2007) 019 [hep-th/0611122].

L. Cornalba, M. S. Costa and J. Penedones, JHEP 0709 (2007) 037 [arXiv:0707.0120 [hep-th]].

R. C. Brower, M. J. Strassler and C. -ITan, JHEP 0903 (2009) 050 [arXiv:0707.2408 [hep-th]].

L. Cornalba, M. S. Costa and J. Penedones, JHEP 1003 (2010) 133 [arXiv:0911.0043 [hep-th]].

R. C. Brower, M. J. Strassler and C. -ITan, JHEP 0903 (2009) 092 [arXiv:0710.4378 [hep-th]].

[7] L. Cornalba, M. S. Costa and J. Penedones, Phys. Rev. Lett. 105 (2010) 072003 [arXiv:1001.1157 [hep-ph]].

[8] M. S. Costa and M. Djuric, Phys. Rev. D 86 (2012) 016009 [arXiv:1201.1307 [hep-th]].

[9] A. Aktas et al. [H1 Collaboration], Eur. Phys. J. C 46 (2006) 585 [hep-ex/0510016]. F. D. Aaron et al. [H1 Collaboration], JHEP 1005 (2010) 032 [arXiv:0910.5831 [hep-ex]].

[10] M. S. Costa, M. Djurić and N. Evans, JHEP 1309 (2013) 084 [arXiv:1307.0009 [hep-ph]].

[11] J. Erdmenger, N. Evans, I. Kirsch and E. Threlfall, Eur. Phys. J. A 35 (2008) 81 [arXiv:0711.4467 [hep-th]].

[12] R. C. Brower, M. Djuric and C. -ITan, JHEP 1209 (2012) 097 [arXiv:1202.4953 [hep-ph]]. 